\title{
Preservation theorems for bounded formulas
}

\author{
Morteza Moniri
}

\begin{abstract}
In this paper we naturally define when a theory has bounded quantifier elimination, or is bounded model complete. We give several equivalent conditions for a theory to have each of these properties. These results provide simple proofs for some known results in the model theory of the bounded arithmetic theories like $\mathrm{CPV}$ and $\mathrm{PV}_{1}$. We use the mentioned results to obtain some independence results in the context of intuitionistic bounded arithmetic. We show that, if the intuitionistic theory of polynomial induction on strict $\Pi_{2}^{b}$ formulas proves decidability of $\Sigma_{1}^{b}$ formulas, then $\mathrm{P}=\mathrm{NP}$. We also prove that, if the mentioned intuitionistic theory proves $\operatorname{LMIN}\left(\Sigma_{1}^{b}\right)$, then $\mathrm{P}=\mathrm{NP}$.
\end{abstract}

2000 Mathematics Subject Classification: 03F30, 03C10, 03F50, 68Q15.

Key words: Bounded Arithmetic, Intuitionistic Logic, Kripke Model, Polynomial Hierarchy, Polynomial Induction, NP, Model Completeness, Quantifier Elimination, Universal Theory.

\section{Preliminaries}

Assume that any theory $T$ we work with below contains $\leqslant$ in its language and that $T$ proves the following basic properties of $\leqslant$. We assume that $T$ proves that $\leqslant$ is reflexive and transitive. We also assume that for all $r$ and $s$, there is a term $t$ so that $T$ proves that $r \leqslant t$ and $s \leqslant t$. We further suppose that, for all terms $t(\bar{x}, y)$ and $r(\bar{x})$, there is a term $s(\bar{x})$ such that $T$ proves $y \leqslant r(\bar{x}) \rightarrow t(\bar{x}, y) \leqslant s(\bar{x})$. Bounded quantifiers and bounded formulas are defined in the usual way. By a $\Sigma_{1}^{b}$ formula we mean a quantifier-free formula prefixed by a bounded existential quantifier. We also call these formulas NP formulas, simultaneously. The reason is that, in this paper, we are mainly concerned with bounded arithmetic theories that use the language of Cook's equational theory PV and have PV as a subtheory. In these theories, the $\Sigma_{1}^{b}$ formulas are exactly the formulas that define the NP relations in the standard model of natural numbers. The $\Pi_{1}^{b}$ and coNP notations will be used in the same way.

A theory is said to be bounded if it is axiomatizable by a set of bounded formulas. To see a proof of the following fact, see e.g. [B2]. 
Fact 1.1 (Parikh's Theorem) Let $T$ be a bounded Theory and $A(\bar{x}, y)$ be a bounded formula. Suppose that $T \vdash \forall \bar{x} \exists y A(x, y)$. Then there is a term $t$ such that $T \vdash \forall \bar{x} \exists y \leqslant$ $t A(\bar{x}, y)$.

The theories $\mathrm{PV}_{1}$ and $\mathrm{CPV}$ of bounded arithmetic are the first-order versions of the equational theory theory PV, and Buss's theory $\mathrm{S}_{2}^{1}$ conservatively extended to the language of $\mathrm{PV}$, respectively. It is known that $\mathrm{PV}_{1}$ is a universal theory, and CPV is $\forall \exists$-conservative over $\mathrm{PV}_{1}$.

The theory IPV is defined as CPV but over intuitionistic logic. CPV is $\forall \exists$-conservative over IPV. For more on IPV, see [CU], [B3], [B4] and [A1]. For a semantical investigation of this theory, see [M1], where Kripke models of intuitionistic bounded arithmetic is studied.

\section{Some model theory for bounded formulas}

In this chapter, we define bounded versions of some well-known notions in model theory, and prove modifications of some well-known theorems of model theory in the context of bounded formulas. These theorems, in some sense, extend the corresponding theorems concerning bounded arithmetic theories which can be proved using the relations between bounded arithmetic and propositional proof complexity, see e.g. [K].

Definition 2.1 Let $T$ be a theory. $T$ has bounded quantifier elimination if any bounded formula is $T$-equivalent to a quantifier-free formula (with the same free variables).

The following proposition can be easily proved via induction on the quantifier complexity of the prenex bounded formulas.

Proposition 2.2 A theory $T$ has bounded quantifier elimination if any $\Sigma_{1}^{b}$ formula is $T$-equivalent to a quantifier-free formula (with the same free variables).

As an example of a theory which has bounded quantifier elimination, one can consider the (first-order) theory PRA (Primitive Recursive Arithmetic). The language of PRA contains a function symbol for each primitive recursive function and the theory has defining axioms for these functions. For more on PRA, see e.g. [A2] and [TD].

The theory PRA is not bounded. There is also a simple way to construct a theory which is bounded and at the same time has bounded quantifier elimination. This method is similar to the way that in the basic model theory one can extend a theory to a theory in a extended language and with quantifier elimination. Let $T$ be a bounded theory in a language $L$. For each bounded $L$-formula $\varphi(\bar{x})$, add a new predicate symbol $R_{\varphi}(\bar{x})$ to $L$ to obtain a language $L^{\prime}$. Also, for each such $\varphi$, add a new axiom $\forall \bar{x}\left(\varphi(\bar{x}) \leftrightarrow R_{\varphi}(\bar{x})\right)$ to $T$ to obtain a new (bounded) theory $T^{\prime}$ in the language $L^{\prime}$. It is easy to see that $T^{\prime}$ has bounded quantifier elimination. 
Definition 2.3 Let $T$ be a theory. A model $M$ of $T$ is called bounded existentially closed if whenever $N$ is a model of $T$ such that $M \subseteq N$, then $M \subseteq_{1}^{b} N$, i.e. for any $\Sigma_{1}^{b}$ formula $\varphi(\bar{x})$ with parameters from $M$, if $N \vDash \varphi(\bar{a})$, then $M \vDash \varphi(\bar{a})$, where $\bar{a} \in M$.

By a well-known result in model theory, any model of a universal theory can be embedded in an existentially closed model of that theory (see e.g. [CK, Lemma 3.5.7]). As a consequence, any model of PV can be embedded in a bounded existentially closed model of PV. In such a model, the extended Frege propositional proof system EF is complete, see [K, Corollary 15.3.10 and Theorem 15.3.12].

Definition 2.4 A theory $T$ is called bounded model complete if whenever $M \subseteq N$ are models of $T$, then $M \subseteq_{1}^{b} N$.

As an example of a theory which is bounded model complete, one can consider the theory PA (Peano Arithmetic). By the famous MRDP theorem, any bounded formula in PA is equivalent to a $\exists$-formula, and so, if $M \subseteq N$ are models of $\mathrm{PA}$, then $N$ is a bounded elementary extension of $M$ (see e.g. [HP]).

The following theorem is similar to a famous result in model theory characterizing model complete theories (see e.g [CK, Theorem 3.5.1]).

Theorem 2.5 Let $T$ be a bounded theory. The following are equivalent.

(1) $T$ is bounded model complete.

(2) Every model of $T$ is a b.e.c. model of $T$.

(3) For any $\Sigma_{1}^{b}$ formula there is a $T$-equivalent $\Pi_{1}^{b}$ formula.

(4) For any bounded formula there is a $T$-equivalent $\Pi_{1}^{b}$ formula.

Proof We only give the proof for the case (1) implies (3). The other parts are straightforward and similar to the ones for the unbounded version of this theorem. Let $\exists x \leqslant t \varphi(x, \bar{w})$ be a $\Sigma_{1}^{b}$ formula. By the assumption and a basic result in model theory characterizing universal formulas (see e.g. [Ho, Theorem 5.4.4]), there is a universal formula $\forall y \psi(y, \bar{w})$ such that $T \vdash \forall y \psi(y, \bar{w}) \leftrightarrow \exists x \leqslant t \varphi(x, \bar{w})$. From $T \vdash \forall y \psi(y, \bar{w}) \rightarrow$ $\exists x \leqslant t \varphi(x, \bar{w})$, we have $T \vdash \exists y \exists x \leqslant t(\psi(y, \bar{w}) \rightarrow \varphi(x, \bar{w}))$. Now, using the fact that $T$ is a bounded theory, by Parikh's theorem (Fact 1.1), there is a term $s$ such that $T \vdash \exists y \leqslant s \exists x \leqslant t(\psi(y, \bar{w}) \rightarrow \varphi(x, \bar{w}))$. So $T \vdash \forall y \leqslant s \psi(y, \bar{w}) \rightarrow \exists x \leqslant t \varphi(x, \bar{w})$. Therefore, $T \vdash \forall y \leqslant s \psi(y, \bar{w}) \leftrightarrow \exists x \leqslant t \varphi(x, \bar{w})$.

Corollary 2.6 Let $T$ be a bounded theory which is bounded model complete. Then $T$ is $\forall \Sigma_{1}^{b}$-axiomatizable.

Proof Using the fact that $T$ is a bounded theory and Theorem 2.5 (4), one can see that the class of models of $T$ is closed under union of chains. So, by a well-known result 
in model theory, $T$ is $\forall \exists$-axiomatizable. Now Parikh's theorem guarantees that $T$ is $\forall \Sigma_{1}^{b}$-axiomatizable.

Corollary 2.7 If $\mathrm{PV}_{1}$ is not equal to $\mathrm{CPV}$, then there exist models $\mathrm{M}$ and $\mathrm{N}$ of $\mathrm{CPV}$ such that $M \subseteq N$ but not $M \subseteq_{1}^{b} N$.

Proof If $\mathrm{PV}_{1}$ is not equal to $\mathrm{CPV}$, then $\mathrm{CPV}$ is not $\forall \Sigma_{1}^{b}$-axiomatizable.

NOTE The above result can also be proved via some known results in bounded arithmetic as follows. We refer to $[\mathrm{K}]$ for the proofs of all mentioned facts. If $\mathrm{PV}_{1}$ is not equal to $\mathrm{CPV}$, then $\mathrm{PV}_{1}$ does not prove $\mathrm{P}=\mathrm{NP}$, since $\mathrm{PV}_{1}$ proves polynomial induction on the quantifier-free formulas. So, $\mathrm{CPV} \nvdash \mathrm{P}=\mathrm{NP}$ as $\mathrm{CPV}$ is a $\forall \exists \Delta_{1}^{b}$-conservative extension of $\mathrm{PV}_{1}$ and $\mathrm{P}=\mathrm{NP}$ is a $\forall \exists \Delta_{1}^{b}$ sentence. Therefore, CPV $\nvdash \mathrm{NP}=$ coNP because $\mathrm{CPV} \vdash \mathrm{P}=\mathrm{NP} \cap$ coNP. So, there is a model of CPV in which the extended Frege system is not complete. Hence, it is not the case that any extension of the mentioned model is $\Sigma_{1}^{b}$ elementary.

\section{Some intuitionistic consequences}

In this section we prove some independence results in the context of intuitionistic bounded arithmetic using the results proved in Chapter 2. Our proofs are based on Kripke model theory for these theories. In the context of intuitionistic bounded arithmetic, where quantifier-free formulas are decidable, Kripke models are normal, i.e. the interpretation of $=$ in each world is true equality, and the accessability relation is substructure. For more on Kripke models of intuitionistic bounded arithmetic, we refer the reader to [B3] and [M1].

Cook and Urquhart [CU] proved that if the theory IPV proves the principe PEM of excluded middle for $\Sigma_{1}^{b}$ formulas, then $\mathrm{P}=\mathrm{NP}$. Below, we prove a similar result for a stronger theory.

Recall that the instance of the length-minimization LMIN with respect to a distinguished free variable $x$ on a formula $\varphi(x)$ (which may have more free variables) is the universal closure of the formula

$$
\exists x \varphi(x) \rightarrow\left[\varphi(0) \vee \exists x\left(\varphi(x) \wedge\left(\forall z \leqslant\left\llcorner\frac{x}{2}\right\lrcorner\right) \neg \varphi(z)\right)\right] .
$$

By a strict $\Pi_{2}^{b}$ formula, denoted $s \Pi_{2}^{b}$, we mean a formula of the form

$\forall x \leqslant t \exists y \leqslant s \varphi(x, y)$, where $\varphi$ is a quantifier-free formula.

Recall that the theory $S_{2}^{2}$ proves polynomial induction on all $\Pi_{2}^{b}$ formulas, see [B1] for the definition of the hierarchy of bounded arithmetic formulas and the mentioned result. 
Theorem 3.1 If the intuitionistic theory of BASIC $+\operatorname{PIND}\left(s \Pi_{2}^{b}\right)$ proves $\operatorname{PEM}\left(\Sigma_{1}^{b}\right)$, then $\mathrm{S}_{2}^{2} \vdash \mathrm{P}=\mathrm{NP}$.

Proof First we show that if $M \subseteq N$ are models of $\mathrm{S}_{2}^{2}$, then the two node tower can be considered as a Kripke model of $\operatorname{BASIC}+\operatorname{PIND}\left(s \Pi_{2}^{b}\right)$. For this, assume that $\psi(x)$ is a $s \Pi_{2}^{b}$ formula with possible parameters from $M$ (and with $x$ as the only free variable). Using the definition of forcing, one can easily see that $\psi(a)$, for $a \in M$, is forced in $M$ if and only if $\psi(a)$ is satisfied in $M$ and $N$. Assume for the purpose of a contradiction, that $\operatorname{PIND}(\psi(x))$ is not forced in $M$. So $\psi(0)$ and $\forall x\left(\psi\left(\left\llcorner\frac{x}{2}\right\lrcorner\right) \rightarrow \psi(x)\right)$ are forced in $M$, but $\forall x \psi(x)$ is not forced in $M$. So $\forall x \psi(x)$ is not satisfied in $M$ since it should be satisfied in $N$ as forcing and satisfaction are equivalent in $N$. Now, using LMIN on the $\Sigma_{2}^{b}$ formula $\neg \psi(x)$ which is available by [B1, Theorem 2.8.17], there exists an $a \in M$ such that $M \not \models \psi(a)$ and for any $b \in M$ with the condition $b \leqslant\left\llcorner\frac{a}{2}\right\lrcorner, M \vDash \psi(b)$. In particular, $M \vDash \psi\left(\left\llcorner\frac{a}{2}\right\lrcorner\right)$. So, $M \Vdash \psi\left(\left\llcorner\frac{a}{2}\right\lrcorner\right)$. Therefore, by $M \Vdash \forall x\left(\psi\left(\left\llcorner\frac{x}{2}\right\lrcorner\right) \rightarrow \psi(x)\right)$, we have $M \Vdash \psi(a)$, contradiction.

So, if the intuitionistic theory of $\operatorname{BASIC}+\operatorname{PIND}\left(s \Pi_{2}^{b}\right)$ proves $\operatorname{PEM}\left(\Sigma_{1}^{b}\right)$, then the twonode Kripke model forces $\operatorname{PEM}\left(\Sigma_{1}^{b}\right)$, and so one can easily see that $M \subseteq_{1}^{b} N$. Hence, we proved that the relation between any two models $M \subseteq N$ of $\mathrm{S}_{2}^{2}$ is $\Sigma_{1}^{b}$-elementary extension. Now use Theorem 2.5 and the fact $\mathrm{CPV} \vdash \mathrm{P}=\mathrm{NP} \cap \operatorname{coNP}$.

The above result implies that, if $\mathrm{P} \neq \mathrm{NP}$, then $\mathrm{BASIC}+\operatorname{PIND}\left(s \Pi_{2}^{b}\right) \nvdash_{i} \operatorname{PIND}\left(\Pi_{2}^{\mathrm{b}+}\right)$. The reason is that, as mentioned in $[\mathrm{Ha}]$, Cook proved that the intuitionistic theory of BASIC $+\operatorname{PIND}\left(\Pi_{2}^{b+}\right)$ proves decidability of $\Sigma_{1}^{b}$ formulas. Here, $\Pi_{2}^{b+}$ denotes the class of positive $\Pi_{2}^{b}$ formulas. A formula is positive if it does not contain $\neg$ and $\rightarrow$.

It is known that $S_{2}^{2}$ proves the scheme length-minimization for $\Sigma_{2}^{b}$ formulas (see [B1, Theorem 2.8.17]). Here we show that even $\operatorname{LMIN}\left(\Sigma_{1}^{\mathrm{b}}\right)$ is not derivable in the intuitionistic theory of BASIC $+\operatorname{PIND}\left(s \Pi_{2}^{b}\right)$, unless $\mathrm{P}=\mathrm{NP}$. In [M2], it is proved that a weak form of $\operatorname{LMIN}\left(\sum_{1}^{\mathrm{b}}\right)$ is not derivable in IPV under the assumption that $\mathrm{PV}_{1}$ is not equal to CPV (and so, unless the polynomial hierarchy collapses by [KPT]).

Theorem 3.2 If the intuitionistic theory of BASIC $+\operatorname{PIND}\left(s \Pi_{2}^{b}\right)$ proves $\operatorname{LMIN}\left(\Sigma_{1}^{b}\right)$, then $\mathrm{S}_{2}^{2} \vdash \mathrm{P}=\mathrm{NP}$.

Proof Assume that $\mathrm{S}_{2}^{2} \nvdash \mathrm{P}=\mathrm{NP}$. So, there exist models $\mathrm{M}$ and $\mathrm{N}$ of $\mathrm{S}_{2}^{2}$ such that $M \subseteq N$ but not $M \subseteq_{1}^{b} N$. Let $\sigma$ be an $L(M)$-sentence which is $\Sigma_{1}^{b}$ and satisfied in $N$ but not in $M$. Let $K$ be the Kripke model of BASIC $+\operatorname{PIND}\left(s \Pi_{2}^{b}\right)$ obtained by putting $N$ above $M$ (see prove of the above Theorem). Let $\varphi(x)$ be the $\Sigma_{1}^{b}$ formula $x=2 \vee \sigma$. We show that $K$ does not force the instance of LMIN on $\varphi(x)$. It is easy to see that $M$ (resp. $N$ ) forces $\varphi(a)$, for $a \in M$, if and only if $M$ (resp. $N$ ) satisfies $\varphi(a)$.

So we have $M \nVdash \varphi(0), M \nVdash \varphi(1)$, and $M \Vdash \varphi(2)$. Moreover, $M \nVdash \neg \varphi(0)$ and 
$M \nVdash \neg \varphi(1)$. Therefore $K \nVdash \operatorname{LMIN}(\varphi(\mathrm{x}))$.

Acknowledgements I would like to thank the referee for useful suggestions. This research was in part supported by a grant from IPM (No. 84030114).

\section{References}

[A1] J. Avigad, Interpreting Classical Theories in Constructive Ones, Journal of Symbolic Logic, 65 (2000) 1785-1812.

[A2] J. Avigad, Saturated Models of Universal Theories, Annals of Pure and Applied Logic 118 (2002), 219-234.

[B1] S. R. Buss, Bounded Arithmetic, Bibliopolis, Naples, 1986.

[B2] S. R. Buss, First-Order Proof Theory of Arithmetic, Handbook of proof theory, 79-147, Stud. Logic Found. Math., 137, North-Holland, Amsterdam, 1998.

[B3] S. R. Buss, On Model Theory for Intuitionistic Bounded Arithmetic with Applications to Independence Results, in: Feasible mathematics, eds S. R. Buss and P. J. Scott, 1990, 27-47, Birkhauser.

[B4] S. R. Buss, A Note on Bootstrapping Intuitionistic Bounded Arithmetic, Proof theory (Leeds, 1990), 149-169, Cambridge University Press, Cambridge, 1992.

[CK] C.C. Chang and J. Keisler, Model theory, North-Holland, 1990.

[CU] S. A. Cook and A. Urquhart, Functional Interpretations of Feasibly Constructive Arithmetic, Annals of Pure and Applied Logic, 63 (1993), 103-200.

[Ha] V. Harnik, Provably Total Functions of Intuitionistic Bounded Arithmetic, Journal of Symbolic Logic, 57 (1992) 466-477.

[Ho] W. Hodges, A Shorter Model Theory, Cambridge University Press, Cambridge, 1997.

[HP] P. Hájek and P. Pudlák, Metamathematics of First-Order Arithmetic, SpringerVerlag, 1993.

[K] J. Krajíček, Bounded Arithmetic, Propositional Logic, and Complexity Theory, Cambridge University Press, 1995. 
[KPT] J. Krajíček, P. Pudlák and G. Takeuti, Bounded Arithmetic and the Polynomial Hierarchy, International Symposium on Mathematical Logic and its Applications (Nagoya, 1988), Annals of Pure and Applied Logic, 52 (1991) 143-153.

[M1] Morteza Moniri, Comparing Constructive Arithmetical Theories Based on NP-PIND and coNP-PIND, Journal of Logic and Computation, 13 (2003) 881-888.

[M2] Morteza Moniri, Polynomial Induction and Length Minimization in Intuitionistic Bounded Arithmetic, Mathematical Logic Quarterly, 51 (2005) 73-76.

[TD] A. S. Troelstra and D. van Dalen, Constructivism in Mathematics, vol. I, NorthHolland, 1988.

\section{ADDRESS:}

Department of Mathematics,

Shahid Beheshti University, Evin,

Tehran, Iran. AND:

Institute for Studies in

Theoretical Physics and Mathematics (IPM),

P.O. Box 19395-5746,

Tehran, Iran.

Email: ezmoniri@ipm.ir AND m-moniri@cc.sbu.ac.ir 